\title{
3D Quantification of Zebrafish Brain Vascular Architecture of Light Sheet Fluorescence Microscopy Datasets
}

Elisabeth Kugler ${ }^{1,2,3}$, James Frost ${ }^{1,4}$, Vishmi Silva ${ }^{1}$, Karen Plant ${ }^{1,2}$, Karishma Chhabria ${ }^{1,2}$, Timothy Chico ${ }^{1,2,3}$, Paul Armitage ${ }^{1,3}$

${ }^{1}$ Department of Infection, Immunity and Cardiovascular Disease, The University of Sheffield, United Kingdom. ${ }^{2}$ The Bateson Centre, University of Sheffield., United Kingdom. ${ }^{3}$ Insigneo Institute for in silico Medicine, University of Sheffield., United Kingdom. ${ }^{4}$ Hull York Medical School, University of York., United Kingdom

\section{Abstract Text}

Background: Zebrafish transgenic lines and light sheet fluorescence microscopy (LSFM) allow unrivalled insights into vascular development in vivo and in 3D. The vascular architecture can be used to provide useful insights into physiological status. However, assessment of the vasculature still relies on individual visual assessment rather than objective quantification. Thus, an image analysis pipeline is required to allow data assessment in 3D robustly and sensitively, while being able to handle LSFM data.

Aim: The aim of this work is to produce an image analysis pipeline that allows quantification of the zebrafish brain vasculature in 3D.

Methods: Image acquisition of anaesthetised transgenic zebrafish embryos from 2-to-5dpf $T g(k d r l: H R A S-m C h e r r y) s 916$ was performed using a Z.1 Zeiss LSFM (TJAC Home Office Project Licence 70/8588). The image analysis software Fiji was used for all analysis steps: enhancement (Sato filter), segmentation (Otsu thresholding), vascular volume (measurement of vascular voxels), registration (manual landmark- or automatic object-based rigid registration), surface extraction (Canny edge), skeletonization (3D thinning), diameters (combination of Euclidean Distance Maps and skeleton), 
length (number of skeleton voxels), branching points (skeleton analysis), and complexity (Sholl analysis)

All steps were integrate into a single Macro, including a GUI [1] (github.com/ElisabethKugler/ZFVascularQuantification).

The quantification workflow was applied to 16 datasets using morpholinos (MO) and pharmacological manipulations (morpholinos for jagged-1a, jagged-1b, dll4, notch1b, ccbe1; pharmacological manipulations of vascular endothelial growth factor inhibition, Notch inhibition, nitric oxide synthase inhibition, Wnt inhibition, Wnt activation, F-actin polymerization inhibition, Myosin II inhibition, osmotic pressure increase, and membrane rigidity decrease)

Results: Robust vascular segmentation[2] allowed extraction of vascular volume and was the foundation for successful landmark- or object-based inter-sample registration, which was found to be robust and applicable from 2-to-5 days post fertilization (dpf). Averaging of registered embryos enabled the extraction of regions of similarity, with greatest similarity found in peripheral vessels in the dorsal cerebral vasculature. We

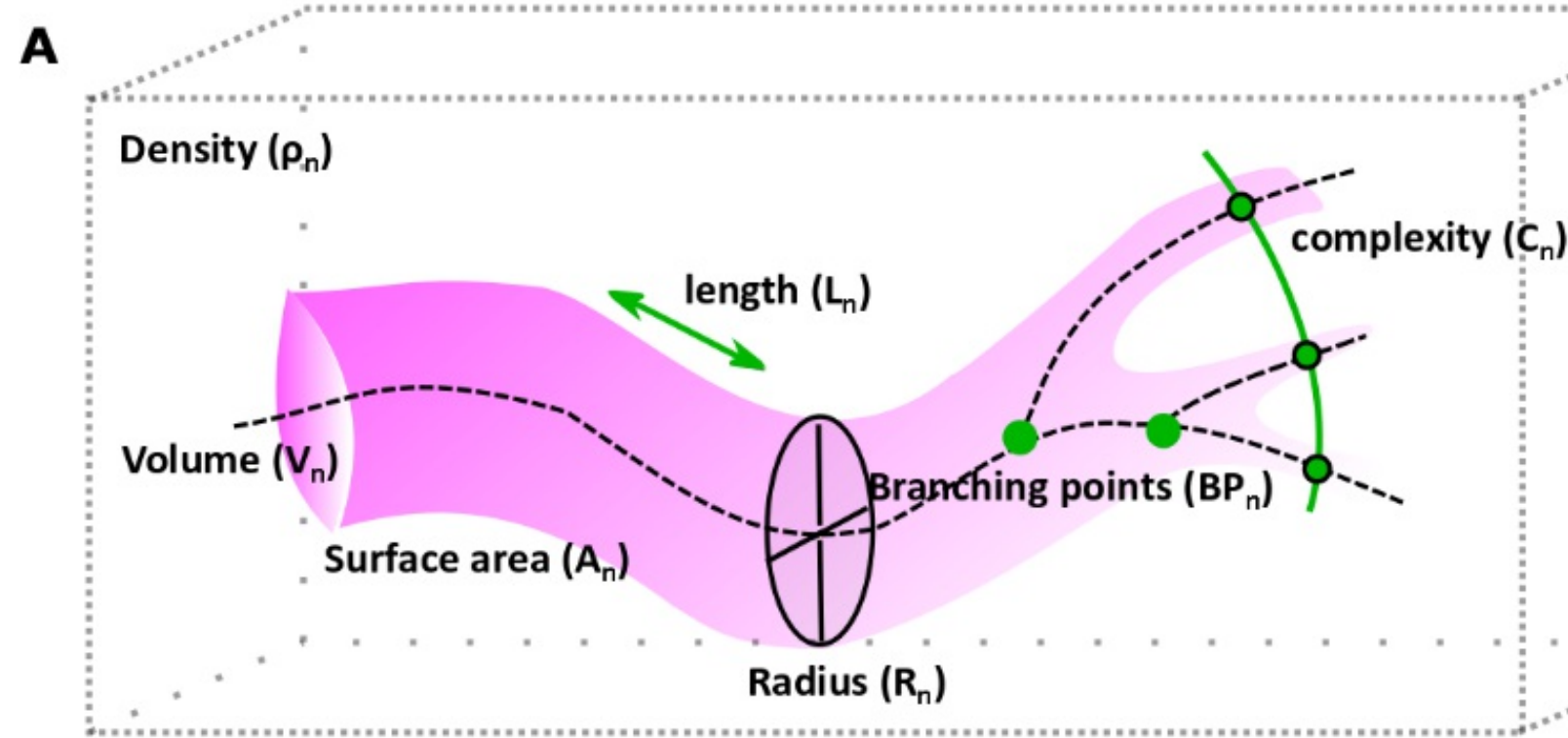

B

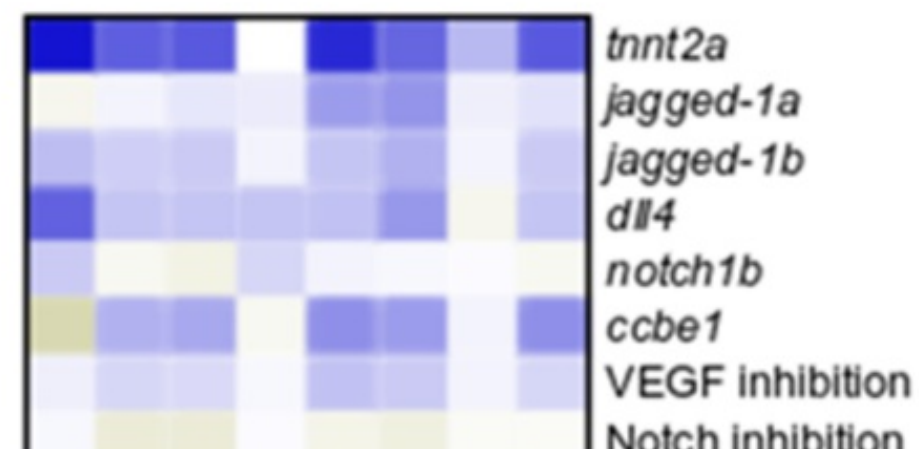



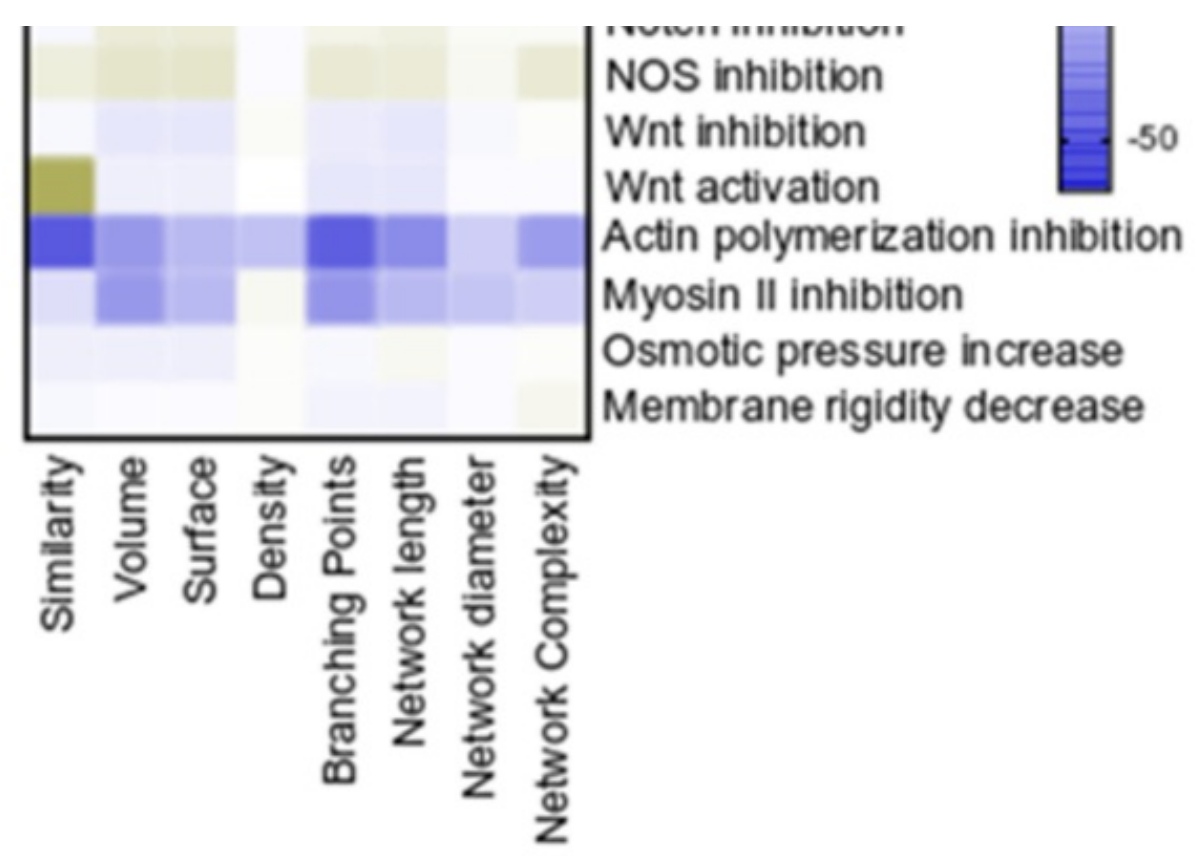

C

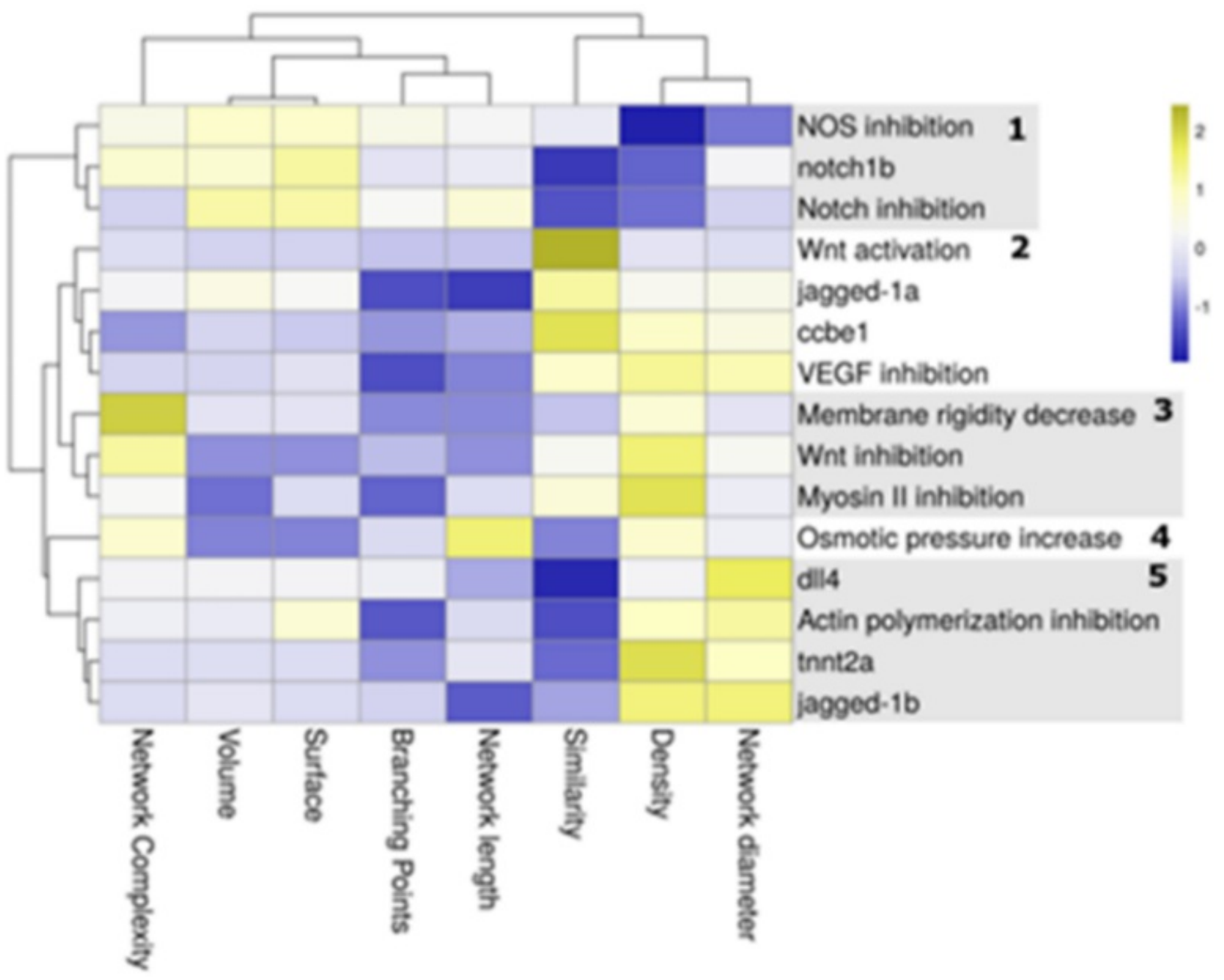

applied our quantification approach to sixteen datasets, and extracted mean differences between controls and treated/MO groups assessed (Fig.1A). Cluster analysis on the examined datasets identified five clusters (Fig.1B), including (1) inhibitors of anti- 
angiogenic factors, (2) inhibitors of angiogenic factors, (3) factors inducing cellular changes, (4) osmotic pressure, and (5) factors with severe angiogenic defects (Fig.1C).

Fig.1(A) Parameters quantified. (B) Quantification of eight vascular parameters, performed for sixteen different datasets. (C) Cluster analysis of these data revealed five main clusters.

Conclusion: Together, we present the first quantification approach to assess the whole 3D cerebrovascular architecture in zebrafish, allows extraction of biologically relevant information, and provides novel insights into vascular biology, 